\title{
Analysis of the spatial distribution between successive earthquakes in aftershocks series
}

\author{
Elisaveta Marekova
}

Plovdiv University "Paisiy Hilendarski”, Department of Experimental Physics, Plovdiv, Bulgaria

\author{
Article history \\ Received June 23, 2014; accepted September 24, 2014. \\ Subject classification: \\ Seismology, Earthquake interaction and probability, General and miscellaneous.
}

\section{ABSTRACT}

The earthquake spatial distribution is being studied, using catalogs for different recent aftershock series. The quality of the available data, taking into account the completeness of the magnitude, is examined. Based on the analysis of the catalogs, it was determined that the probability densities of the inter-event distance distribution collapse into a single curve when the data were rescaled. The collapse of the data provides a clear illustration of aftershock-occurrence self-similarity in space.

\section{Introduction}

Ever since the observations of Omori [Omori 1894] temporal clustering of earthquakes has been seen as a remarkable feature of seismic appearance. Spatial clustering has also been evaluated [Kagan and Knopoff 1980] and together with the Omori and Gutenberg-Richter laws [Gutenberg and Richter 1965], it has become groundwork for probabilistic tools for defining time-dependent seismic hazard [Ogata 1988, Reasenberg and Jones 1989, Gerstenberger et al. 2005].

The catalogs of earthquakes, both contemporary and historical, allow drawing several important conclusions on the mechanisms of seismicity by describing of the inter-event properties, i.e. by separation of successive events in time and space. Many studies have attempted to describe the type of distribution of these inter-event properties, using global and regional seismic catalogs [Bak et al. 2002, Corral 2004, Davidsen and Goltz 2004, Davidsen and Paczuski 2005, Corral 2006b, Touati et al. 2009].

The distribution $D(\Delta t)$ of the inter-time $\Delta t$ between two successive events is a suitable quantity for describing of the temporal organization of seismicity. In an analogous manner, the distribution $D(\Delta r)$ of the distances $\Delta r$ between epicenters for successive earthquakes provides a convenient tool for describing the spatial organization.

Both distributions have been the subject of much research in recent years [Bak et al. 2002, Mega et al. 2003,
Corral 2004, Yang et al. 2004, Corral 2005a, Davidsen and Paczuski 2005, Lindman et al. 2005, Lippiello et al. 2005, Livina et al. 2005, Corral 2006a, Corral 2006b, Saichev and Sornette 2006, Lippiello et al. 2007, Bottiglieri et al. 2010, Lippiello et al. 2012]. These distributions show universal behavior, independent from the considered region or the magnitude range, as described by Corral [2004, 2006b]; Saichev and Sornette [2006]; Davidsen and Paczuski [2005]. E.g., for the region of South California, marked with intensive observations during the last decades, results have been reported, according to which both the inter-event distances or "jumps" between the earthquake epicenters [Davidsen and Paczuski 2005, Corral 2006b], and the inter-event times, or return times [Bak et al. 2002, Corral 2004, Corral 2006b], show statistical distributions in the form of a power-law. This is a manifestation of a complex spatiotemporal (self-) organization of seismicity [Saichev and Sornette 2006].

It should be noted that most of the authors focus on one of these properties only, i.e., either on the interevent separation distance or on return times. Some argue, however, that the understanding of the kinematics of earthquake realization suggests a link between the inter-event properties [Batac and Kantz 2013]. While some authors report observing a universal law of return time distribution, obtained by means of rescaling data [Corral 2004, 2006b], others adduce arguments that the spatial scale of the observations is important for the return times distribution [Davidsen and Goltz 2004, Touati et al. 2009].

Touati et al. [2009] describe the visible differences in the inter-event times distributions in regional and global earthquake catalogs: the histogram of the interevent times for the earthquakes in South California shows two different peaks, thus expressing the differences in the waiting times for the correlated (some aftershock series) and the independent events, while the 
global statistic shows a single peak due to the overlapping sequences of different regions. These authors explain their results by means of an epidemic-type aftershock sequence (ETAS) model [Kagan and Knopoff 1981, Ogata 1988, Sornette and Helmstetter 2002].

In Batac and Kantz [2013] an attempt is made to complete the analysis of Touati et al. [2009], using the separation of the distributions of the epicentral distances depending on the corresponding time intervals between successive earthquakes. The analysis is performed in accordance with the fact that the spatiotemporal clustering is an established property of seismicity [Omori 1894, Kagan and Knopoff 1980]. Instead of making an attempt to find a fitting function for the inter-event distances and time distributions between successive earthquakes, the study puts an accent on the links between them by means of a simple procedure, whose parameters are derived from the data. The conditional distributions of the earthquake return times, depending on the spatial distribution of events, show that the earthquakes separated by short (long) distances are more likely to be separated by short (long) time intervals. This clearly demonstrates the clustering (separation) of the dependent (independent) events. The interesting thing here is that the different catalogs used by Batac and Kantz [2013], show behavior, already described in the ETAS model [Touati et al. 2009].

\section{Methodology and results}

The sequences of seismic events due to the realization of strong earthquakes (main shocks) and the socalled aftershock series (secondary shocks) are subject of extensive research in seismology. There are many secondary events immediately after a strong earthquake for a relatively short time period. The aftershock sequences are potential sources of information about the physical and mechanical properties of the medium in the focal zone and the processes going on in it. Main characteristics of these sequences are their spatial location as well as the rate at which a sequence weakens with time.

The main objective of this work is the study and analysis of the distribution of distances between successive earthquakes from different aftershock series. The parameters of the considered series are described in Table 1. They include the time of realization and the magnitude of the main shock, as well as the chosen time interval, defining the duration of a series; the number of events in it and the minimum magnitude $\mathrm{M}_{\mathrm{C}}$, over which the sequence is complete, i.e., there are no events omitted at registration. Checking for completeness is carried out by the program Zmap [Wiemer 2001]. The table also includes the depth interval of the considered aftershock series $(\mathrm{H})$. The time duration of the sequences is selected so that it does not exceed

\begin{tabular}{|c|c|c|c|c|c|c|c|}
\hline No. & Earthquake & Date & Period & $M_{\text {main shock }}$ & $\mathbf{M}_{\mathrm{C}}$ & Number of events & $\mathrm{H}[\mathrm{km}]$ \\
\hline 1 & St. Elias,Canada & 28.02 .79 & $28.02 .79-30.03 .79$ & 7.1 & 3.0 & 174 & $0-19$ \\
\hline 2 & Joshua Tree, California & 23.04 .92 & $23.04 .92-28.06 .92$ & 6.1 & 3.0 & 234 & $0-18$ \\
\hline 3 & Landers, California & 28.06 .92 & $28.06 .92-31.12 .93$ & 7.3 & 3.0 & 803 & $0-20$ \\
\hline 4 & Big Bear, California & 28.06 .92 & $28.06 .92-31.12 .93$ & 6.4 & 3.0 & 411 & $0-20$ \\
\hline 5 & Nahanni, Canada & 05.10 .85 & $05.10 .85-20.12 .85$ & 6.6 & 3.1 & 244 & $6-10$ \\
\hline 6 & Andreanof Island '86, Alaska & 07.05 .86 & $07.05 .86-15.06 .88$ & 8.0 & 4.8 & 245 & $18-67$ \\
\hline 7 & Andreanof Island '96, Alaska & 10.06 .96 & $10.06 .96-23.12 .98$ & 7.7 & 4.5 & 179 & $10-60$ \\
\hline 8 & Aegean '81 & 19.12 .81 & $19.12 .81-20.06 .83$ & 7.2 & 3.2 & 261 & $0-35$ \\
\hline 9 & Aegean '82 & 18.01 .82 & $18.01 .82-05.04 .83$ & 6.9 & 3.5 & 77 & $0-72$ \\
\hline 10 & Aegean '83 & 06.08 .83 & $06.08 .83-12.06 .84$ & 6.9 & 3.6 & 100 & $1-47$ \\
\hline 11 & Albania & 15.04 .79 & $15.04 .79-10.10 .79$ & 7.2 & 3.0 & 461 & $0-22$ \\
\hline 12 & Strazica, Bulgaria & 07.12 .86 & $07.12 .86-11.05 .87$ & 5.7 & 2.0 & 123 & $0-25$ \\
\hline 13 & Hector Mine, California & 16.10 .99 & $16.10 .99-01.11 .00$ & 7.1 & 3.0 & 529 & $0-18$ \\
\hline 14 & Northridge, California & 17.01 .94 & $17.01 .94-26.09 .95$ & 6.7 & 3.0 & 451 & $0-23$ \\
\hline 15 & Greece '81 & 24.02 .81 & $24.12 .81-08.10 .82$ & 6.6 & 3.2 & 575 & $0-47$ \\
\hline 16 & Friuli, Italy & 06.05 .76 & $06.05 .76-05.06 .77$ & 6.5 & 3.0 & 277 & $0-58$ \\
\hline
\end{tabular}

Table 1. Description of the studied aftershock series. $M_{\text {main shock }}$ is the main earthquake magnitude; $M_{C}$ is the magnitude of completeness, $\mathrm{H}$ is the depth interval for earthquakes in series. 
Knopoff's window, depending on the magnitudes of the major earthquakes [Gardner and Knopoff 1974].

A modern trend in recent times is to visualize a wide range of natural systems as networks. The networks are complex web-like structures, consisting of nodes and branches [Albert and Barabasi 2002]. Such structures are also used for the description of seismic events and aftershock series in particular [Baiesi and Paczuski 2005]. All pairs of successive events are considered for the purpose in a wide spatiotemporal window. This approach is used to define appropriate metrics, which could determine the level of connection between the successive events, taking into account the established statistical properties of seismicity. This procedure allows identifying the aftershock groups by means of a quantitative measure.

This paper offers a different approach. The spatial properties of several predetermined aftershock series are studied. Only the successive events from the sequences are used for this purpose.

The spatial distribution analysis was carried out following Marekova [2014]. Due to the usually higher relative and absolute error for the depth of earthquakes, only epicenters from seismicity catalogs were considered.

The distances between the epicenters (jumps) of the $i$-th earthquake and the previous in time event, $(i-1)$, were calculated from the catalog. A FORTRAN program was developed, intending to calculate distances between points on the Earth's surface. For this purpose, conversion of the geographic coordinates $(\varphi, \lambda)$ into geocentric $\left(\varphi^{\mathrm{G}}, \lambda^{\mathrm{G}}\right)$ was performed, in order to take into account the ellipsoid shape of the Earth:

$$
\begin{gathered}
\varphi^{\mathrm{G}}=\varphi+0.00339466 \sin (2 \varphi) \\
\lambda^{\mathrm{G}}=\lambda
\end{gathered}
$$

Then the distance $\Delta^{\circ}$ (in degrees) was defined by the formula:

$$
\begin{aligned}
& \cos \left(\Delta^{\circ}\right)= \\
& \sin \varphi_{1}^{\mathrm{G}} \sin \varphi_{2}^{\mathrm{G}}+\cos \varphi_{1}^{\mathrm{G}} \cos \varphi_{2}^{\mathrm{G}} \cos \left(\lambda_{2}^{\mathrm{G}}-\lambda_{1}^{\mathrm{G}}\right)
\end{aligned}
$$

The indexes 1 and 2 were assigned to the coordinates of the pair of events from the series catalog. The results for $\Delta^{\circ}$ were converted into kilometers $d$, one angular degree being equal to approximately $111.1 \mathrm{~km}$.

In this way, a list of the spatial distances $d_{i}$ between successive events is obtained. The range of change of the distances is divided into subintervals of length $\Delta d$. The number of $n_{j}$ values for each subinterval is taken into account and its relative frequency is calculated:

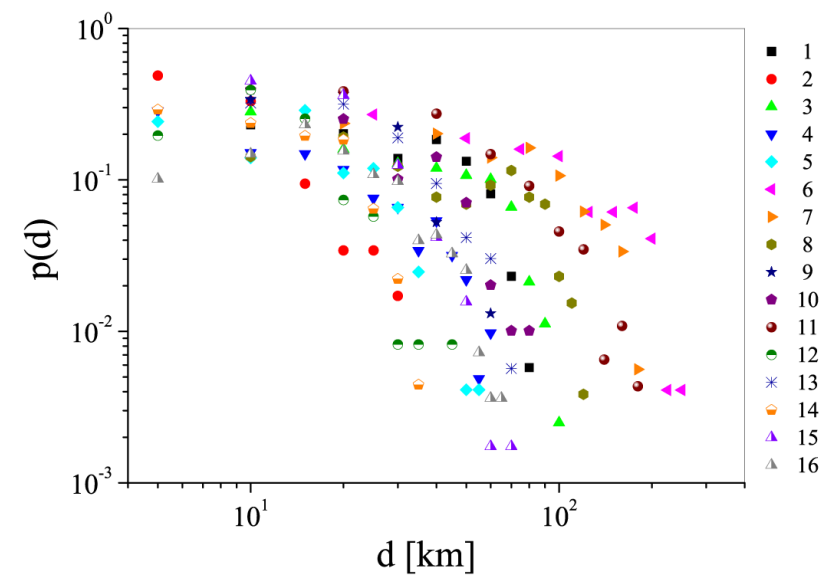

Figure 1. Frequency distribution $p(d)$ of the spatial distances versus distance value $d$, for the epicenters of the earthquakes listed in Table 1 .

$$
p_{j}(d)=\frac{n_{j}(d)}{N}
$$

where $N$ is the total number of shocks in the aftershock series, $j=1, \ldots, k-k$ is the number of subintervals into which each range for the distances is divided.

Figure 1 shows the graphs obtained for the distribution $p(d)$, considering different aftershock series. It can be seen that there is a similarity in the curves - but, the curves are scattered from one another.

I find it more useful to describe the distribution of the inter-event distance by means of a probability density, $P(\Delta d)$, defined as

$$
P(\Delta d)=\frac{\operatorname{Prob}\left(d<d^{\prime} \leq d+\Delta d\right)}{\Delta d}
$$

where Prob denotes probability, and $\Delta d$ describes the bins over which the probability density is calculated.

It is straightforward to estimate $P(\Delta d)$ from $p_{j}(d)$ as

$$
P_{j}(\Delta d)=\frac{p_{j}(d)}{\Delta d}=\frac{n_{j}(d)}{\Delta d N}
$$

where $N$ is the total number of earthquakes.

The probability density removes the dependence of the size distribution on $\Delta d$, as well as extension of the spatial region under analysis.

The curves show in Figure 1 can be transformed into coordinates, rescaled as follows.

The $x$-axis is divided by $L$ and the $y$-axis is multiplied by $L$, i.e.

$$
\begin{gathered}
x=\frac{\Delta d}{L} \\
y=L^{*} P(\Delta d)
\end{gathered}
$$

where $L$ is the maximum distance, obtained for the investigated series.

Pre-selected series of aftershocks are used in the presented study. The duration and the spatial distribu- 
tion of the aftershock series are selected so that they correspond to the ones defined by the Knopoff's window - one of the methods for selecting aftershocks. According to this method all events, which have occurred in the radius of $\mathrm{R}(\mathrm{M}) \mathrm{km}$ with respect to the main quake or for less time than the interval of $\mathrm{T}(\mathrm{M})$ days, are considered to be aftershocks of this particular quake. $R(M)$ and $\mathrm{T}(\mathrm{M})$ depend on the magnitude of the main quake.

The maximum distance $L$ used in this study is obtained only for successive quakes in the series. The observed maximum values of $L$ for the various series are in most cases smaller than those calculated by Gardner and Knopoff [1974] for outlining the aftershock region.

The equation, describing the relationship between the magnitude of the main quake and the obtained maximum distances between successive quakes is:

$L(M)=L_{0} 10^{\alpha M}$, where $L_{0}=0.05 \pm 0.05$ and $\alpha=0.46 \pm 0.06$.

This result complies with the result of Bottiglieri et al. [2010]. The defined maximum distance $L$ can be used as a characteristic dimension, related to the aftershock region, what has been done analogically in the above cited paper.

The process of rescaling causes a change in the curves of Figure 1, presented in Figure 2.

It can be seen that considering the inter-event distances distribution, the data collapses when rescaled.

Attempts for an approximation of the distance distributions between successive quakes from aftershock series by means of various functions have been made. Curd et al. [2000], for example, suggest for this purpose gamma and Weibull distributions.

It is shown in Marekova [2014] that distributions of the inter-event distances are successfully approximated by the so-called beta-distribution, using for this

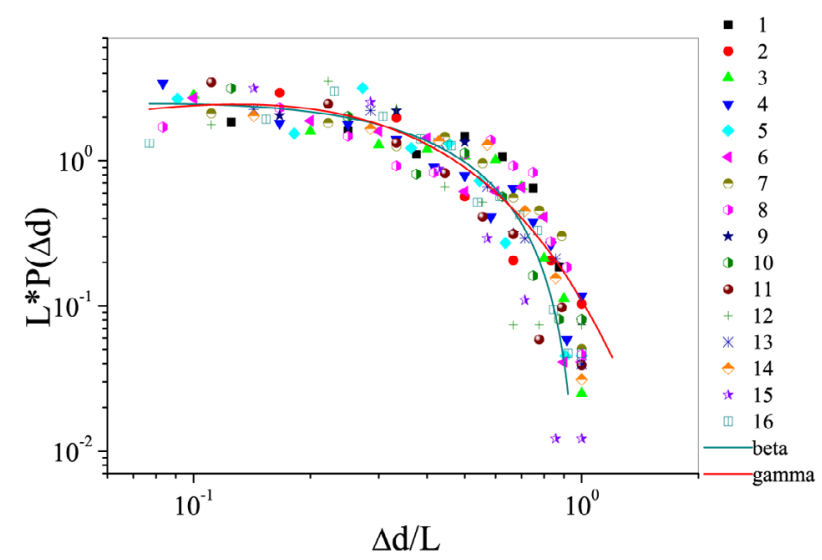

Figure 2. Probability density for the rescaled data: $x=\Delta d / L ; y=$ $L^{*} P(\Delta d)$ from catalogs, listed in Table 1 . The green continuous line is a beta distribution fit and the red continuous line - a gamma distribution fit. purpose data from regional catalogs.

The Beta distribution is a power function of the variable $x$ and its reflection $(1-x)$ with shape parameters $\alpha>0$ and $\beta>0$ (real numbers):

$$
f(x)=\frac{x^{\alpha-1}(1-x)^{\beta-1}}{B(\alpha, \beta)}
$$

where $B(\alpha, \beta)$ represents the beta function, which appears as a normalization constant to ensure that the total probability integrates to unity; and $0<x<1$.

By approximation function (9) was used in the form:

$$
f(x)=a x^{\alpha-1}(1-x)^{\beta-1}
$$

Parameters in (9a) have the values:

$$
\begin{aligned}
& a=4.47 \pm 1.09 \\
& \alpha=1.17 \pm 0.10 \\
& \beta=3.02 \pm 0.27
\end{aligned}
$$

Figure 2 also shows the model curve for the case of data approximation by gamma distribution. The function is applied in the present paper in the following form [Corral 2005b]:

$$
f(x)=a(b x)^{\gamma-1} \exp (-b x)
$$

where $a, b$, and $\gamma$ are the parameters:

$$
\begin{aligned}
& a=6.24 \pm 0.38 \\
& b=5.13 \pm 0.57 \\
& \gamma=1.67 \pm 0.14
\end{aligned}
$$

In many publications this function appears to be appropriate for fitting the distributions of the time intervals between successive earthquakes [Wang and Kuo 1998, Corral 2004, Corral 2005b, Corral 2006a, Corral 2006b, Hainzl et al. 2006, Apostol et al. 2008, Corral 2009, Marekova 2012].

As it can be seen from Figure 2, beta distribution describes the data better than gamma distribution. In gamma distribution the deviation of the real data from the model curve is within the big values of $x-$ for $x>0.7$; at smaller values of $x$ the two curves do not differ significantly.

For the same series the distribution of distances $r$ between the main shock and the subsequent secondary earthquakes has also been studied. The series Landers, Big Bear, Nahanni, Hector mine, Andreanof Island ' 86 and Friuli have been selected. All of them are characterized by a large number of events.

After using the methodology described in this paper the results show that in case of mixing all distributions of the distances $r$, an analogical result is not ob- 


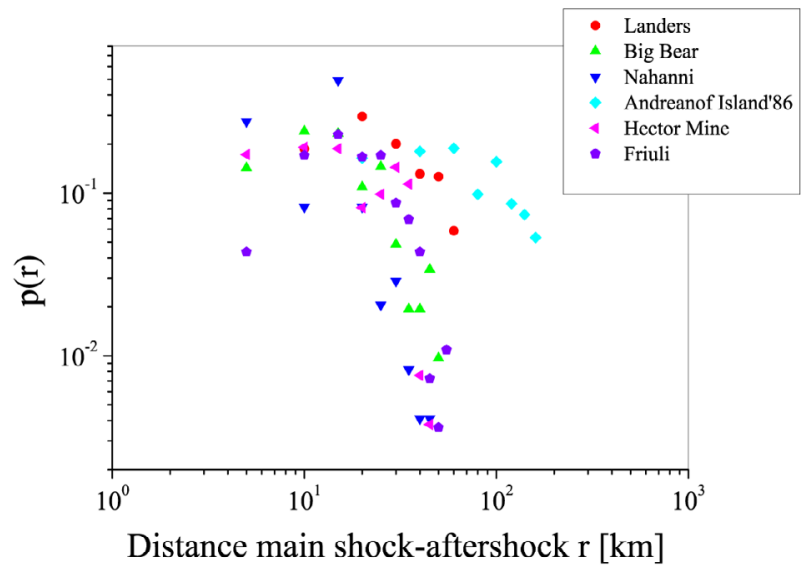

Figure 3. Frequency distribution $p(d)$ versus main shock-aftershock distance $r$, for the selected earthquakes.

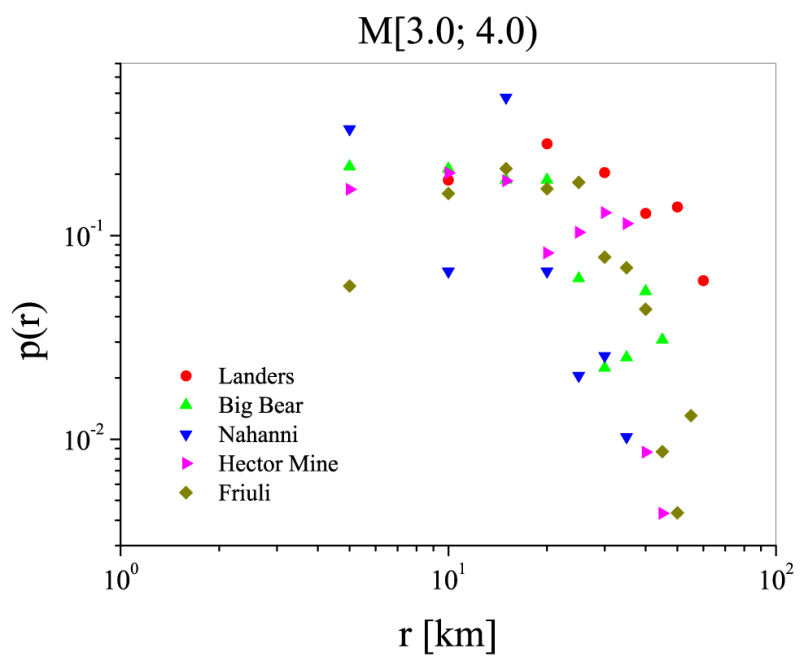

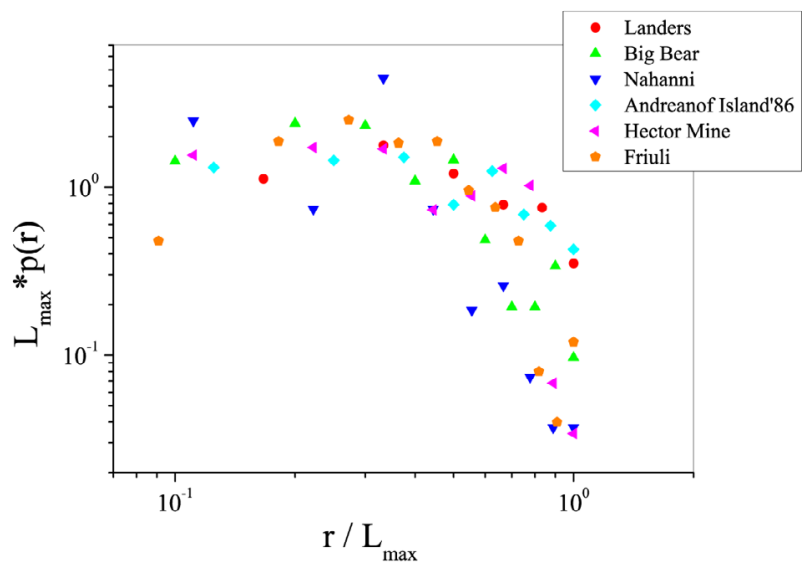

Figure 4. Probability density for the rescaled data: $x=r / L_{\max } ; y=$ $L_{\max } * P(r)-r$ main shock-aftershock distance, for the selected earthquakes.

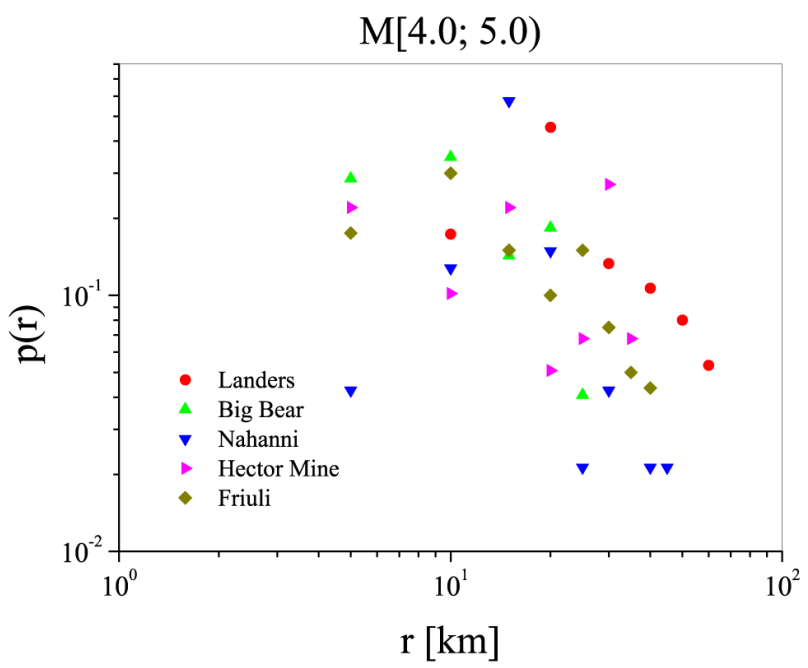

Figure 5. Frequency distribution $p(d)$ versus main shock-aftershock distance $r$, for the selected earthquakes in varius magnitude intervals.
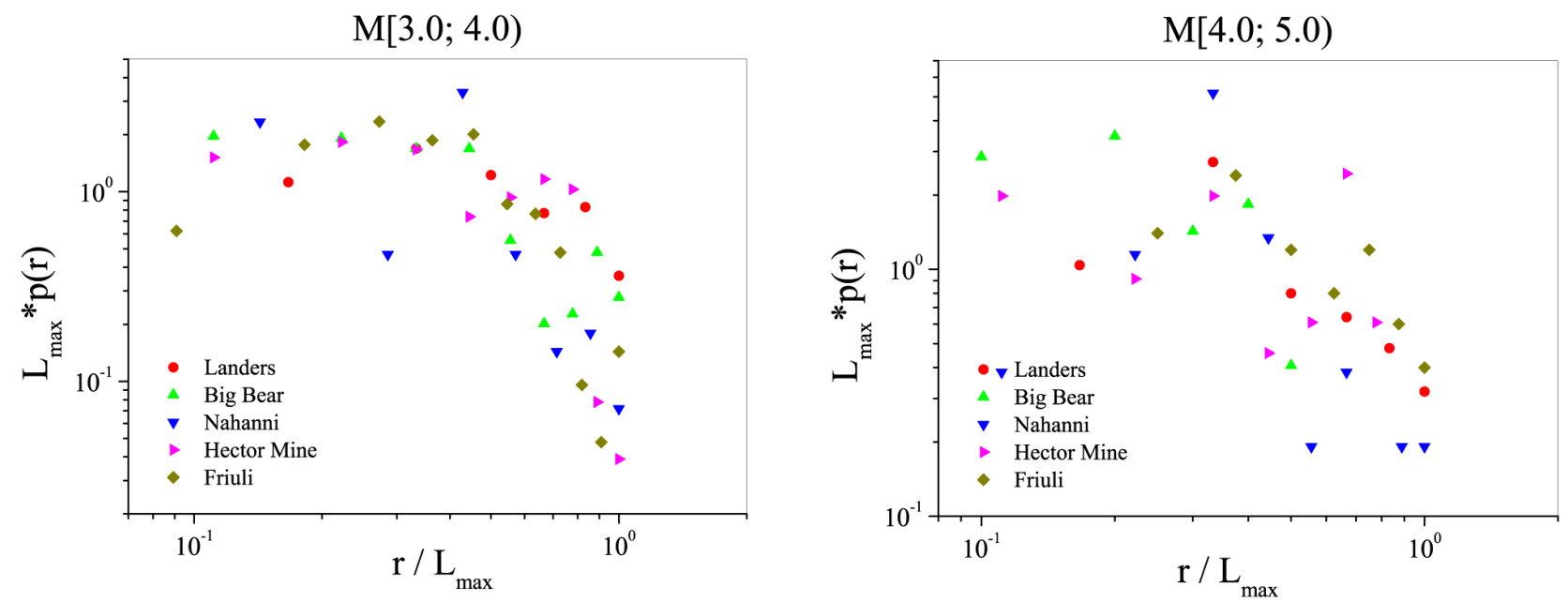

Figure 6. Probability density for the rescaled data: $x=r / L_{\max } ; y=L_{\max } * P(r)-r$ main shock-aftershock distance, for the selected earthquakes in various magnitude intervals.

served. The curves do not collapse not only in the case of frequency distribution $p(r)$, but also after re-scaling (Figures 3 and 4 ). In these cases $L_{\max }$ is the distance to the most remote aftershock in the corresponding series.

Series with different magnitude ranges have been selected for some of the aftershock sequences. Mixing the data on the corresponding magnitude intervals, analogical to the one carried out in Bottiglieri et al. [2010], Lippiello et al. [2009], does not lead to the collapse of the data again (Figures 5 and 6). 
Therefore, following the idea of Lippiello et al. [2009] and Felzer and Brodsky [2006] the present study has been complemented with a study of the spatial density of the aftershocks. Linear density has been analyzed in this case, i.e., the number of aftershocks per unit of length.

Because of the difference in the way of formation of the series in this work and in the cited papers, a different approach is also used in this study. For the series, pointed out above the analysis has first been made for a number of time intervals of the aftershock activity for the whole interval, for the 1 st day, for the first 10 days, for the first 100 days (Figure 7). Choosing a shorter interval, e.g. 30 minutes, as in Lippiello et al. [2009] and Felzer and Brodsky [2006], leads to a great reduction of the number of quakes in the series, what makes the results unconvincing. It should be noted that all studied aftershock series comprise events with a definite threshold of completeness, which in most cases is $M_{C}=3.0$.

The data from all time intervals can be approximated by a power law:

$$
p(r)=c r^{-\delta_{T}}
$$

where $c$ is a constant, varying in dependence on the number of aftershocks. The values of the exponent $\delta_{T}$ change for the different sequences:

- Landers - from 2.1 to 2.3;

- Big Bear - from 2.0 to 2.2;

- Hector Mine - from 1.4 to 2.3;

- Nahanni - from 2.5 to 3.0;

- Andreanof Island ' 86 - from 1.5 to 1.8;

- Friuli - from 3.5 to 4.0.

For some of the series fitting is carried out at $r \geq 10$ $\mathrm{km}$. Besides, the data about some of the time intervals cannot be approximated by a power law - e.g. for the 1 st day for Big Bear and Friuli.

The other study of the above aftershock series is in dependence on the magnitudes of the earthquakes. Magnitude intervals M[3.0; 4.0) and $[4.0 ; 5.0)$ have been selected together with the data about the whole series, which are with $M_{C} \geq 3.0$. The data for $M \geq 5.0$ have not been analyzed since their number is small. The only exception is the series of the earthquake Andreanof Island ' 86 . It has a minimum magnitude of completeness $\mathrm{M}_{\mathrm{C}} \geq 4.8$. The intervals in it are correspondingly $\mathrm{M}[4.8$; $5.5)$ and $[5.0 ; 6.5)$, together with the whole series [4.8; 8.0] (Figure 8).

In these cases the data about the linear density as a function of the distance "main shock - secondary quake" can also be fitted by a power law with an exponent $\delta_{M}$, and in most cases this is done at $r \geq 10 \mathrm{~km}$.

The values of the exponent $\delta_{M}$ change as follows:
- Landers - from 1.1 to 1.3;

- Big Bear - from 2.2 to 2.3;

- Hector Mine - from 1.9 to 2.5;

- Nahanni - from 2.6 to 3.0;

- Andreanof Island '86 - from 1.51 to 1.53;

- Friuli - from 2.6 to 3.2 .

This time again for two of the series the data cannot be fitted by the power law. These are Big Bear for $\mathrm{M}[4.0 ; 5.0)$ and Andreanof Island ' 86 for $[5.0 ; 6.5)$.

Aftershocks are defined by their cluster properties both in time and in space. Temporal clustering follows the well-defined Omori law.

The spatial distribution of the aftershocks is more complex and not fully explained yet. On the one hand, it is clear that many of the aftershocks occur along the surface of the fault or in its immediate vicinity. Aftershock grouping along or near the destruction caused by the main shock may reflect the local concentration of stress on asperities, serving to stop destruction and close the fault. On the other hand, aftershocks occur also away from the fault line due to various trigger mechanisms, the simplest of which is the stress transfer and the increase of the Coulomb stress [Stein 1999].

The investigation of linear density $\rho(r)$, where $r$ is the epicentral distance between each aftershock and its related mainshock, represents a useful tool to discriminate between triggering by static or dynamic stress [Felzer and Brodsky 2006, Lippiello et al. 2009].

In these papers, $\rho(r)$ decay asymptotically as $r^{-\mu}$, where $\mu \simeq 1.4$ to 1.9 [Lippiello et al. 2009] and $\mu \simeq 1.4$ [Felzer and Brodsky 2006].

Here I show that the linear density exhibits a power law behavior, but the obtained exponents are more scattered. The basic differences with this study and previous analyses is that aftershocks have been classified according to the mainshock magnitude and distances in a different manner. Therefore it is necessary to carry out joint research on these properties of aftershock series, which will be the subject of future investigation.

Seismic events are complex spatial-temporal phenomena [Turcotte 1993, Sornette 1999, Bak et al. 2002, Pavlos et al. 2007, Vecchio et al. 2008, Sornette and Werner 2009], since they occur in clusters and exhibit scale-invariant characteristics. The most typical description in such cases is the division of the earthquakes into foreshocks, main quakes and aftershocks. Very often during the analysis of their properties in space and time power laws are used.

Nowadays widely used to describe such complex phenomena is the definition of the so-called fractal dimension [Mandelbrot 1982]. The concept of fractal dimension takes central place in understanding the chaotic picture of seismicity, especially for defining the level of 


\section{Landers earthquake}

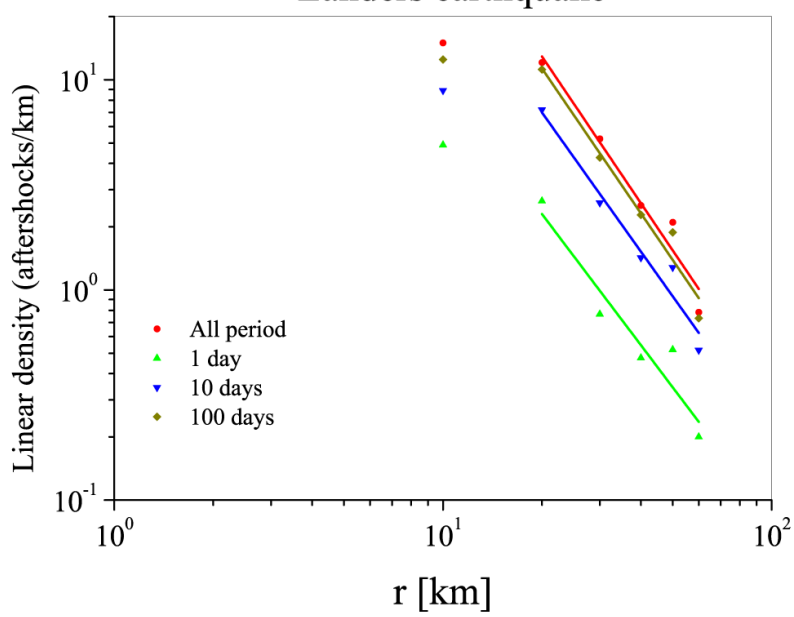

Nahanni earthquake

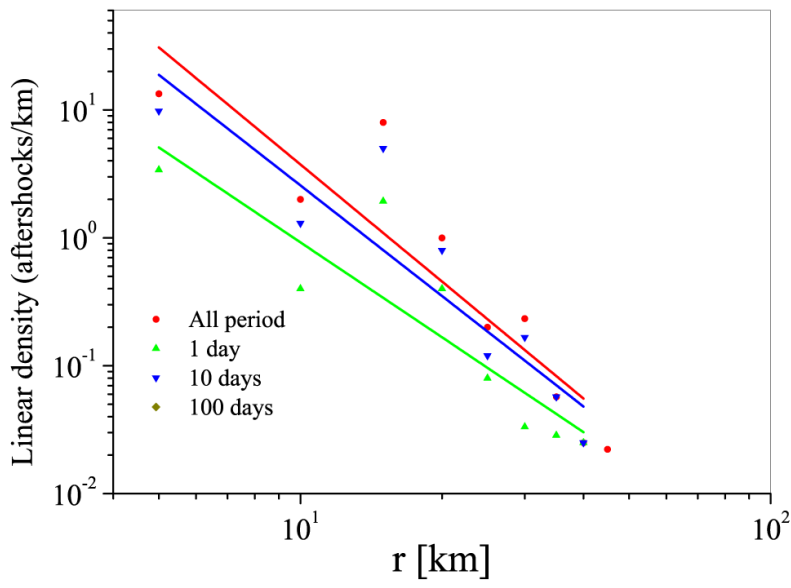

Hector Mine earthquake

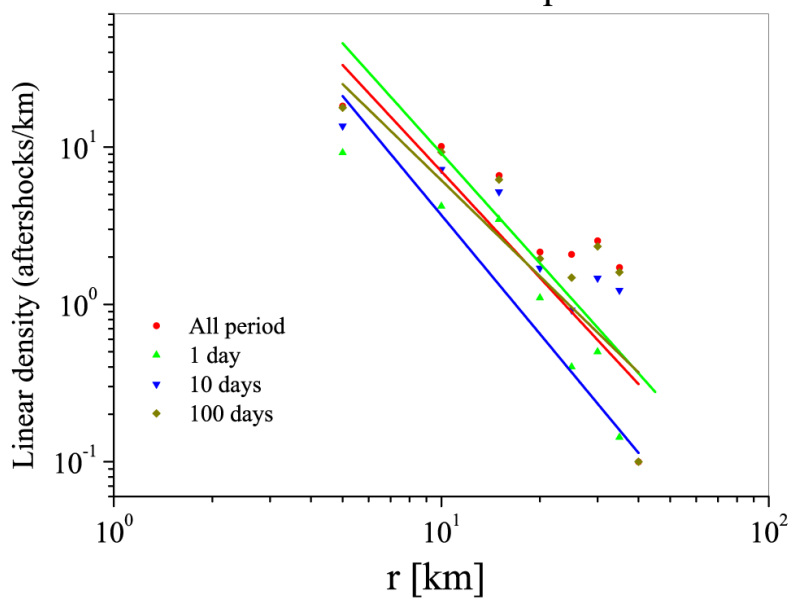

Big Bear earthquake
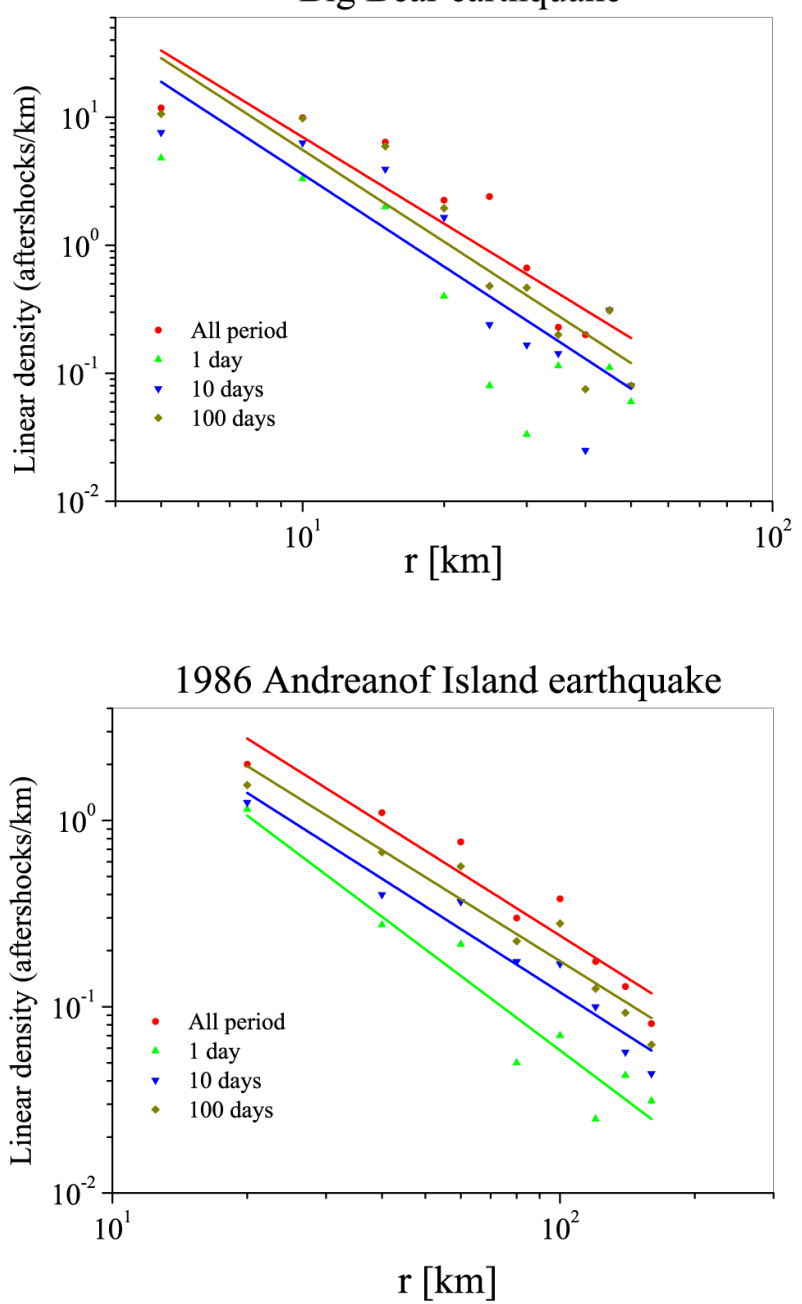

Friuli earthquake

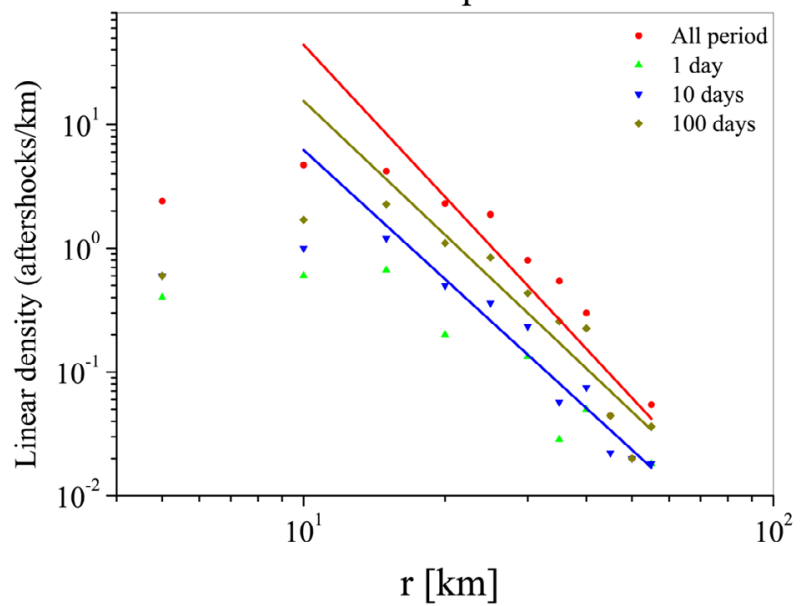

Figure 7. Linear density versus main shock-aftershock distance $r$, for the selected earthquakes in various time intervals.

clusterization in the earthquakes' spatial distribution. The value of the fractal dimension gives a quantitative measure of the process of grouping for the epicenters / hypocenters of the "main" events, both for the active regions [Kagan and Knopoff 1980, De Rubeis et al. 1993, Tosi et al. 2004, Tosi et al. 2008] and for the aftershock regions [Robertson et al. 1995, Nanjo and Nagahama 2000, Nanjo and Nagahama 2004].
In the works of Bak et al. [2002] the structure of seismicity in space, time and in magnitude is revealed, placing all earthquakes on the same base - without making difference between main and secondary quakes [Bak et al. 2002, Christensen et al. 2002]. Thus seismicity is regarded as a single process.

The above researchers propose a new scaling law, outlining the framework for assessing the likelihood of 
Landers earthquake
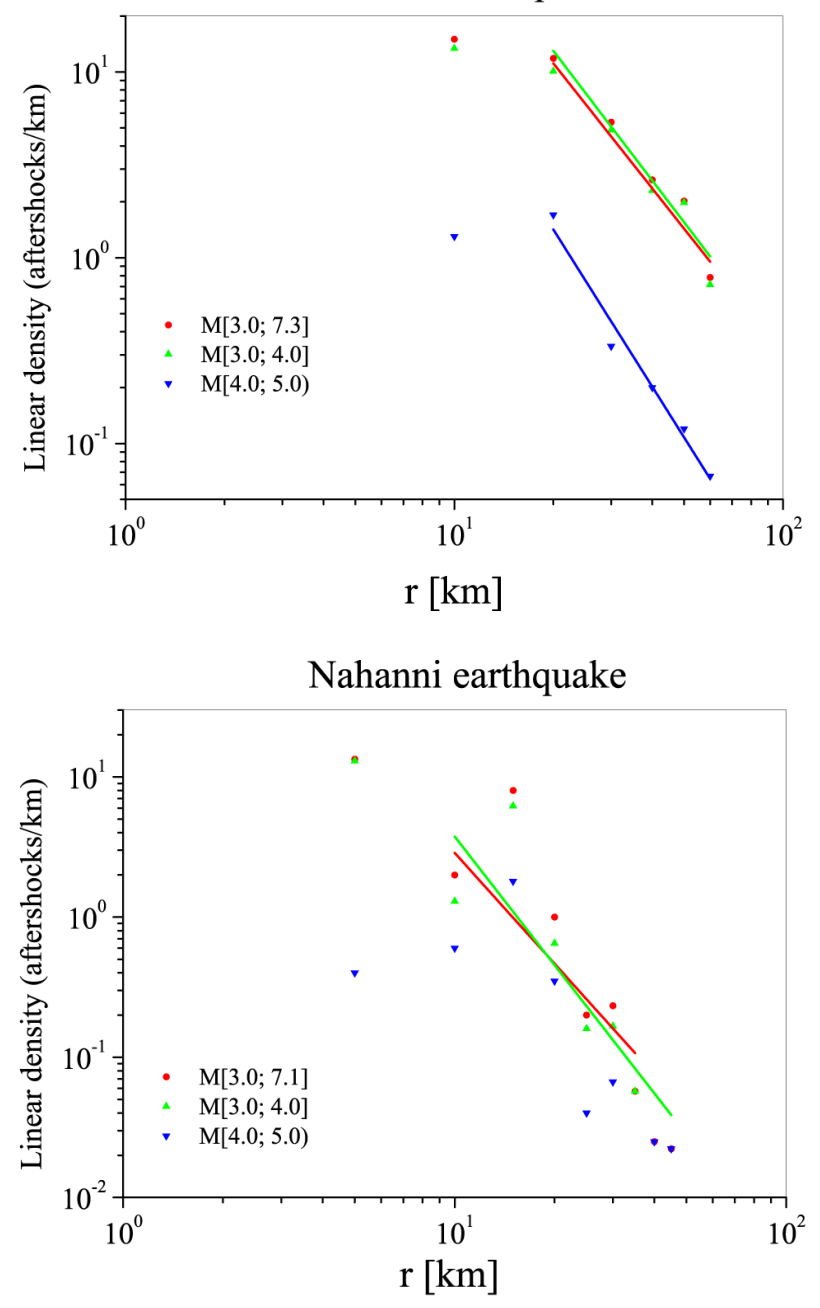

Hector Mine earthquake

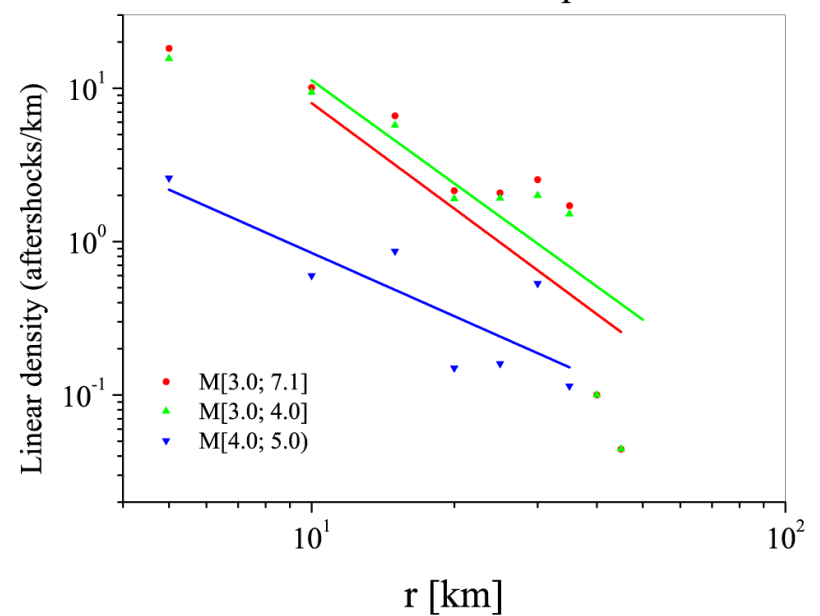

Big Bear earthquake
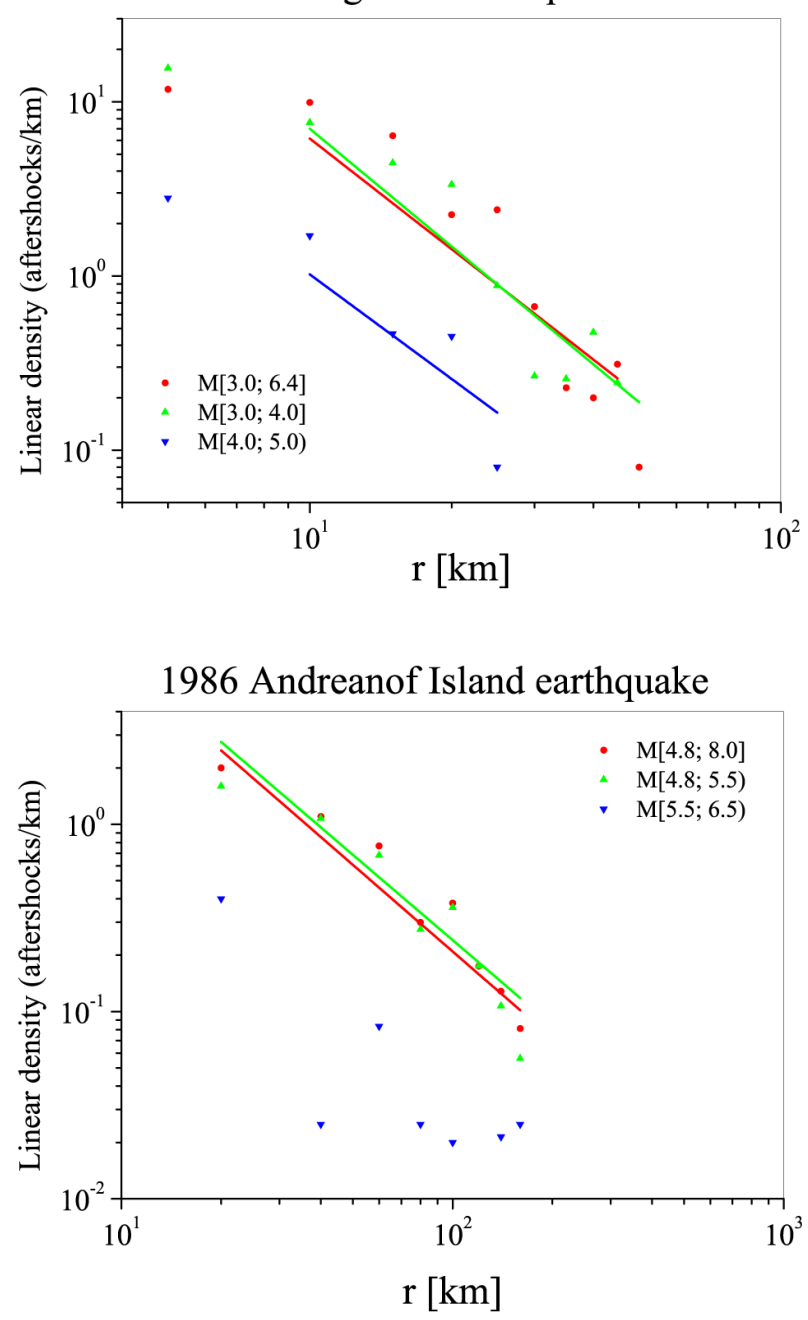

Friuli earthquake

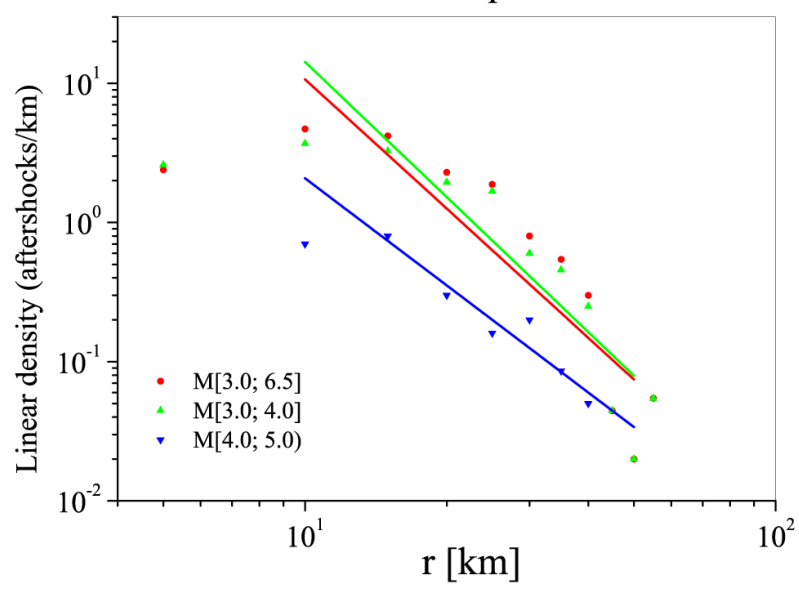

Figure 8. Linear density versus main shock-aftershock distance $r$, for the selected earthquakes in various magnitude intervals.

earthquakes in a given region for a given threshold magnitude. The law shows that the seismic events occur in hierarchically related clusters, which overlap other spatially separated clusters for sufficiently big time intervals and regions. For sufficiently small regions and temporal scales only single related groups can be selected. The aftershocks are usually described as related events, which occur after a strong seismic event. This definition is widely used in order to classify the catalogs, containing seismic data, as catalogs of aftershocks and catalogs of main shocks. Omori's law is based on this classification of the earthquakes and it usually suggests that the aftershocks are due to mechanisms other than the ones that caused the main events.

Gardner and Knopoff [1974] assume that an earthquake can be classified as an aftershock if it belongs to 
a cluster of related events; it can be regarded as a main quake if it belongs to a series of "isolated" random events with Poisson statistics. However, nowadays a reliable scientific method for differentiating whether an earthquake is an aftershock or a main quake does not exist, since, according to this definition, the categorization depends on the considered scales for length, time and magnitude. The proposed law puts together the law of Gutenberg-Richter, Omori's law and the law describing the fractality of the fault systems.

In many other publications after Bak et al. [2002] when spatial-temporal properties are concerned, all seismic events are considered together [Corral 2003, Corral 2004, Davidsen and Goltz 2004, Lindman et. al. 2005, Corral 2005b, Shcherbakov et al. 2005, Corral 2006a, Corral 2006b, Saichev and Sornette 2006, Shcherbakov et al. 2006].

Following the studies described above, I have considered the spatial distributions of both aftershock series and earthquakes from declusterized series [Marekova 2014]. The purpose is to have the idea of considering seismic events as a single process developed in more than one way.

\section{Conclusions}

This paper presents an investigation of the distributions of distances between consecutive earthquakes from different aftershock series. It has been established that when these distributions are rescaled, all curves converge in one. The rescaled data are well fitted by a beta distribution.

The obtained results are similar to those, reported in Marekova [2014]. It can be noted that the distributions of the distances between successive earthquakes in declusterized series of regional seismicity [Marekova 2014] and aftershock series, separated by the conventional spatiotemporal windows, are analogical. They are well approximated by beta distribution. It gives reason to consider that the spatial distributions of the earthquakes are similar irrespective of their separation in series of major (independent) and secondary (dependent) events. The differences can be seen only in the extension of the regions in which they are distributed. In the process of rescaling the distributions, as it can be seen from Figure 2 and analogical figures in Marekova [2014], these differences disappear. This provides a clear illustration of earthquake-occurrence self-similarity in space.

Considering the distance distributions it is important that the data collapse, which is a proof of a critical process, since only these exhibit this type of collapse, is known as scaling in critical phenomena. This analysis shows that earthquakes are a phenomenon of SOC [Bak et al. 1987, Bak 1997, Kanamori and Brodsky
2004]. This is strikingly reminiscent of the collapse onto a single curve [Varotsos et al. 2005, 2011] of the order parameter scaled distributions deduced from the seismicity in various regions worldwide when they are analyzed in a new time domain termed natural time [Varotsos et al. 2001, Sarlis et al. 2008], which has the privilege to extract the maximum information possible from a given time series [Abe et al. 2005].

Both in distance distributions for declusterized sequences, and in aftershock series there is no tendency for the real distributions to exceed the values given by the model curve; there is an excess of short inter-event distances. Such a tendency appears when all earthquakes from regional catalogs are considered on the same base, without dividing them into foreshocks, main quakes and aftershocks.

In most studied cases, regions where these points exist are relatively large in area. Since during data selection aftershock series have not been removed, it is possible that the availability of an increased number of short distances between the earthquakes is due to them. In smaller sized regions, such an increased number of small distances is not observed, as the distances between the aftershocks in them and the distances between the independent events will be comparable.

The division into series of independent and dependent events shows that they have similar spatial distributions. Therefore the observed deviations could be due to some more specific manifestation of seismic activity, though they could also be explained by some disadvantages in the methodology of the study or the properties of the analyzed catalogs.

The separate analysis of both the inter-event distances and times between successive earthquakes allows marking important properties of seismicity. Such an analysis is made both for regional seismicity, wherein all earthquakes are placed on the same base and for declusterized sequences and aftershock series separately. The results will be complemented and extended if a joint study on space-time properties of seismic activity, similar to that in Batac and Kantz [2013] is carried out.

\section{References}

Abe, S., N.V. Sarlis, E.S. Skordas, H.K. Tanaka and P.A. Varotsos (2005). Origin of the Usefulness of the Natural-Time Representation of Complex Time Series, Phys. Rev. Lett., 94, 170601; doi:10.1103/PhysRevLett.94.170601.

Albert, R., and A.L. Barabási (2002). Statistical mechanics of complex networks, Rev. Mod. Phys., 74, 47-97.

Apostol, B.F., L.C. Cune and M. Apostol (2008). Scaling and universal power laws in time series of seismic 
events, Rom. Journ. Phys., 53 (3-4), 593-600.

Baiesi, M., and M. Paczuski (2004). Scale Free Networks of Earthquakes and Aftershocks, Phys. Rev. E 69, 066106; doi:10.1103/PhysRevE.69.066106.

Bak, P., C. Tang and K. Wiesenfeld (1987). Self-organized criticality: an explanation of $1 / \mathrm{f}$ noise, Phys. Rev. Lett., 59, 381-384.

Bak, P. (1997). How Nature Works. The Science of Selforganized Criticality, Copernicus, New York, and Oxford University Press, Oxford, 214 pp.

Bak, P., K. Christensen, L. Danon and T. Scanlon (2002). Unified Scaling Law for Earthquakes, Phys. Rev. Lett., 88, 178501 (4 pp.); doi:10.1103/PhysRevLet. 88.178501 .

Batac, R.C., and H. Kantz (2013). Spatiotemporal clustering and separation in regional earthquakes, arXiv: 1307.1609v1 [physics.geo-ph].

Bottiglieri, M., L. de Arcangelis, C. Godano and E. Lippiello (2010). Multiple-time scaling and universal behaviour of the earthquake inter-event time distribution, Phys. Rev. Lett., 104, 158501; doi:10.1103/ PhysRevLett.104.158501.

Christensen, K., L. Danon, T. Scanlon and P. Bak (2002). Unified Scaling Law for Earthquakes, Proc. Natl. Acad. Sci. USA, 99, 2509-2513; www.pnas.org_cgi_ doi_10.1073_pnas.012581099.

Corral, A. (2003), Local distributions and rate fluctuations in a unified scaling law for earthquakes, Phys. Rev. E 68, 035102(R).

Corral, A. (2004). Long-term clustering, scaling, and universality in the temporal occurrence of earthquakes, Phys. Rev. Lett., 92, 108501 (4 pp.); doi:10. 1103 / PhysRevLett.92.108501.

Corral, A. (2005a). Renormalization-Group Transformations and Correlations of Seismicity, Phys. Rev. Lett., 95, 028501 (4 pp.); doi:10.1103/PhysRevLett. 95.028501 .

Corral, A. (2005b). Time-decreasing hazard and increasing time until the next earthquake, Phys. Rev. E 71, 017101.

Corral, A. (2006a). Dependence of earthquake recurrence times and independence of magnitudes on seismicity history, Tectonophysics, 424, 177-193; doi:10.1016/j.tecto.2006.03.035.

Corral, A. (2006b). Universal Earthquake-Occurrence Jumps, Correlations with Time, and Anomalous Diffusion, Phys. Rev. Lett., 97, 178501 (4 pp.); doi:10. 1103 / PhysRevLett.97.178501.

Corral, A. (2009). Statistical tests for scaling in the interevent times of earthquakes in California, Int. J. Mod. Phys. B, 23 (28-29), 5570-5582.

Curd, S.R., P.W. Burton, C.A. Davenport, E. Sokos and G.A. Tselentis (2000). Statistical analyses of after- shocks and swarms associated with active faults in central Greece: inter-event separation as an indicator of seismotectonic setting?, In: Proceedings of the XXVII General Assembly of the European Seismological Commission, Lisbon, 215-220.

Davidsen, J., and C. Goltz (2004). Are seismic waiting time distributions universal?, Geophys. Res. Lett., 31, L21612 (4 pp.); doi:10.1029/2004GL020892.

Davidsen, J., and M. Paczuski (2005). Analysis of the Spatial Distribution Between Successive Earthquakes, Phys. Rev. Lett., 94, 048501 (4 pp.); doi:10. 1103 / PhysRevLett.94.048501.

De Rubeis, V., P. Dimitriu, E. Papadimitriu and P. Tosi (1993). Recurrent patterns in the spatial behaviour of Italian seismicity revealed by the fractal approach, Geophys. Res. Lett., 20, 1911-1914.

Felzer, K.R., and E.E. Brodsky (2006). Decay of aftershock density with distance indicates triggering by dynamic stress, Nature, 441, 735-737.

Gardner, J.K., and L. Knopoff (1974). Is the sequence of earthquakes in Southern California with aftershocks removed, Poissonian?, B. Seismol. Soc. Am., 64 (5), 1363-1367.

Gerstenberger, M.C., S. Wiemer, L.M. Jones and P.A. Reasenberg (2005). Real-time forecasts of tomorrow's earthquakes in California, Nature, 435 (7040), 328-331.

Gutenberg, B., and C.F. Richter (1965). Seismicity of the Earth, Hafner Pub. Co., New York.

Hainzl, S., F. Scherbaum and C. Beauval (2006). Estimating Background Activity Based on IntereventTime Distribution, B. Seismol. Soc. Am., 96 (1), 313-320; doi:10.1785/0120050053.

Horner, R.B. (1983). Seismicity in the St. Elias region of northwestern Canada and southeastern Alaska, B. Seismol. Soc. Am., 73 (4), 1117-1137 (data for St. Elias earthquake).

Kagan ,Y., and L. Knopoff (1980). Spatial distribution of earthquakes: the two-point correlation function, Geophys. J. R. Astr. Soc., 62 (2), 303-320.

Kagan, Y., and L. Knopoff (1981). Stochastic synthesis of earthquake catalogues, J. Geophys. Res., 86, 28532869.

Kanamori, H., and E.E. Brodsky (2004). The physics of earthquakes, Rep. Prog. Phys., 67, 1429-1496; doi:10. 1088/0034-4885/67/8/R03.

Lindman, M., K. Jonsdottir, R. Roberts, B. Lund and R. Bodvarsson (2005). Earthquakes descaled: On waiting time distributions and scaling laws, Phys. Rev. Lett., 94 (10), 108501(4); doi:10.1103 / Phys-RevLett. 94.108501.

Lippiello, E., L. de Arcangelis and C. Godano (2005). Memory in self-organized criticality, Europhys. Lett., 
$72,678-682$.

Lippiello, E., C. Godano and L. de Arcangelis (2007). Dynamical scaling in branching models for seismicity, Phys. Rev. Lett., 98 (9), 098501.

Lippiello, E., L. de Arcangelis and C. Godano (2009). Role of Static Stress Diffusion in the Spatiotemporal Organization of Aftershocks, Phys. Rev. Lett., 103, 038501; doi:10.1103/PhysRevLett.103.038501.

Lippiello, E., A. Corral, M. Bottiglieri, C. Godano and L. de Arcangelis (2012). Scaling behavior of the earthquake intertime distribution: influence of large shocks and time scales in the Omori law, Phys Rev E Stat Nonlin Soft Matter Phys., 86 (6 Pt 2), 066119.

Livina, V.N., S. Havlin and A. Bunde (2005). Memory in the occurence of earthquakes, Phys. Rev. Lett., 95, 208501 (4 pp); doi:10.1103/PhysRevLett.95.208501.

Mandelbrot, B. (1982) The fractal geometry of nature, San Francisco: Freeman and Co., 460 pp.

Marekova, E. (2012). Testing a Scaling Law for the Earthquake Recurrence Time Distributions, Acta Geophysica, 60 (3), 858-873; doi:10.2478/s11600-012-0007-y.

Marekova, E. (2014), Analysis of the spatial distribution between successive earthquakes occurred in various regions in the world, Acta Geophysica, 62 (6), 12621282; doi:10.2478/s11600-014-0234-5.

Mega, M.S., P. Allegrini, P. Grigolini, V. Latora and L. Palatella (2003). Power-law time distribution of large earthquakes, Phys. Rev. Lett., 90, 188501.

Nanjo, K., and H. Nagahama (2000). Spatial Distribution of Aftershocks and the Fractal Structure of Active Fault Systems, Pure Appl. Geophys., 157, 575-588.

Nanjo, K., and H. Nagahama (2004). Discussions on fractals, aftershocks, and active faults: diffusion and seismo-electromagnetism, Arab. J. Sci. Eng., 29 (2C), 147-167.

Ogata, Y. (1988). Statistical models for earthquake occurrences and residual analysis for point processes, J. Am. Stat. Assoc., 83, 9-27.

Omori, F. (1894). On aftershocks, Rep. Imp. Earthquake Invest. Comm., 2, 103-139 (in Japanese).

Pavlos, G.P., A.C. Iliopoulos and M.A. Athanasiu (2007). Self Organized Criticality and/ or Low Dimensional Chaos in Earthquake Processes: Theory and Practic in Hellenic Region, Nonlinear Dynamics in Geosciences, 235-259; ISBN: 978-0-387-34917-6.

Reasenberg, P.A., and L.M. Jones (1989). Earthquake Hazard After a Mainshock in California, Science, 243 (4895), 1173-1176; doi:10.1126/science.243.4895. 1173243.

Robertson, M.C., C.G. Sammis, M. Sahimi and A.J. Martin (1995). Fractal Analysis of threedimensional Spatial Distributions of Earthquakes with a Percolation Interpretation, J. Geophys. Res., 100 (B1), 609-620.
Saichev, A., and D. Sornette (2006). "Universal" distribution of interearthquake times explained, Phys. Rev. Lett., 97 (7), 078501 (4 pp.); doi:10.1103/ PhysRevLett.97.078501.

Sarlis, N.V., E.S. Skordas, M.S. Lazaridou and P.A. Varotsos (2008). Investigation of the seismicity after the initiation of a Seismic Electric Signal activity until the main shock, Proceedings of the Japan Academy, Ser. , 84, 331-343.

Shcherbakov, R., G. Yakovlev, D.L. Turcotte and J.B. Rundle (2005). Model for the Distribution of Aftershock Interoccurence Times, Phys. Rev. Lett., 95 (21), 218501(4).

Shcherbakov, R., J.V. Aalsburg, J.B. Rundle and D.L. Turcotte (2006). Correlations in aftershock and seismicity patterns, Tectonophysics, 413, 53-62.

Sornette, D. (1999). Earthquakes: from chemical alteration to mechanical rupture, Physics Report, 313 (50), 237-292; doi:10.1016/S0370-1573(98)00088-X.

Sornette, D., and A. Helmstetter (2002). On the occurrence of finite-time singularities in epidemic models of rupture, earthquakes and starquakes, Phys. Rev. Lett., 89, 158501.

Sornette, D., and M.J. Werner (2009). Statistical Physics Approaches to Seismicity, In: W.H.K. Lee (ed.), Complexity in Earthquakes, Tsunamis, and Volcanoes, and Forecast, In: R.A. Meyers (Editor-in-chief), Encyclopedia of Complexity and Systems Science, 7872-7891, Springer; ISBN: 978-0-387-755888-6, http: / / arXiv.org/abs/0803.3756.

Stein, R.S. (1999). The role of stress transfer in earthquake occurrence, Nature, 402 (N6762), 605-609; doi:10.1038/45144.

Tosi, P., V. De Rubeis, V. Loreto and L. Pietronero (2004). Space-time combined correlation integral and earthquake interactions, Annals of Geophysics, 47 (6), 1849-1854.

Tosi, P., V. De Rubeis, V. Loreto and L. Pietronero (2008), Space-time correlation of earthquakes, Geophys. J. Int., 173, 932-941.

Touati, S., M. Naylor and I.G. Main (2009). Origin and nonuniversality of the earthquake interevent time distribution, Phys. Rev. Lett., 102, 168501 (4 pp.); doi:10.1103/PhysRevLett.102.168501.

Turcotte, D.L. (1993). Fractals and Chaos in Geology and Geophysics, Cambridge University Press, Cambridge, England.

Varotsos, P., N. Sarlis and E. Skordas (2001). Spatiotemporal complexity aspects on the interrelation between Seismic Electric Signals and seismicity, Practica of Athens Academy, 76, 294-321.

Varotsos, P.A., N.V. Sarlis, H.K. Tanaka and E.S. Skordas (2005). Similarity of fluctuations in correlated sys- 
tems: The case of seismicity, Phys. Rev. E 72, 041103(8); doi:10.1103/ PhysRevE.72.041103.

Varotsos, P.A., N.V. Sarlis and E.S. Skordas (2011). Natural ime nalysis: he ew View of Time. Precursory Seismic Electric Signals, Earthquakes and other Complex Time Series, Springer, Heidelberg, 452 pp.; ISBN: 978-3-642-16448-4.

Vecchio, A., V. Carbone, L. Sorriso-Valvo, C. De Rose, I. Guerra and P. Harabaglia (2008). Statistical properties of earthquakes clustering, Nonlinear Proc. Geoph., 15, 333-338.

Yang, X., S. Du and J. Ma (2004). Do Earthquakes Exhibit Self-Organized Criticality?, Phys. Rev. Lett., 92, 228501 (4 pp.); doi:10.1103/ PhysRevLett.92.228501.

Wang, J.-H., and C.-H. Kuo (1998). On the frequency distribution of interoccurrence times of earthquakes, J. Seismol., 2, 351-358; doi:10.1023/ A:10097 74819512 .

Wiemer, S. (2001). A software package to analyze seismicity: Zmap, Seismol. Res. Lett., 72 (2), 373-382; doi: 10.1785 / gssrl.72.3.373, http: / / seismo.ethz.ch/ staff/stefan/IntrotoZMAP6_online.htm.

\section{Data sources for aftershock series:}

Joshua Tree, Landers, Big Bear:

http: / / quake.geo.berkeley.edu/cnss

Andreanof Island 1986:

http: / / giseis.alaska.edu/Seis /

Andreanof Island 1996:

http: / quake.geo.berkeley.edu/cnss

Hector Mine, Northridge:

http: / / www.scecdc.scec.org; or

http:/ / www.ncedc.org/anss /

St. Elias, Canada:

Horner [1983].

Nahanni:

CD from World Data Center-A, Boulder, Colorado

All other data: from personal communication with colleagues.

All original catalogs are included in electronic supplement: file Aftershock series.xls.

\footnotetext{
${ }^{\star}$ Corresponding author: Elisaveta Marekova, Plovdiv University "Paisiy Hilendarski”, Dept. of Experimental Physics, Plovdiv, Bulgaria; email: eligeo@uni-plovdiv.bg, eligeo@yahoo.com. 